\title{
EFEITOS DE MBTA [CLORIDRATO DE N,N-DIETIL-2-(4-METILBENZILOXI) ETILAMINA] NA PRODUTIVIDADE E NA QUALIDADE DOS FRUTOS DA LARANJEIRA 'PÊRA' (Citrus sinensis L. Osbeck) ${ }^{1}$
}

\author{
CHRYZMELINSKI SERCILOTO², PAULO ROBERTO DECAMARGOECASTRO, SILVIOTAVARES
}

RESUMO - O objetivo deste trabalho foi avaliar os efeitos do biorregulador MBTA [cloridrato de N,N-dietil-2-(4-metilbenziloxi) etilamina] aplicado em diferentes épocas e concentrações na produtividade e qualidade dos frutos da laranjeira 'Pêra'. Em duas safras consecutivas, o MBTA foi aplicado em três diferentes concentrações $\left(8 ; 16\right.$ e $\left.32 \mathrm{mg} \mathrm{L}^{-1}\right)$ e em duas diferentes fases fenológicas (25\% e $100 \%$ de flores abertas), em árvores cítricas adultas, utilizando um volume de 7 litros de solução por planta, acompanhado do adjuvante Silwett L-77 0,05\%. Foram amostrados 20 frutos por planta, em quatro diferentes épocas estudadas, para determinar o teor de sólidos solúveis (SS); acidez titulável (AT); quantidade de sólidos solúveis por caixa de 40,8 kg; pH; rendimento de suco; "ratio" (relação SS/AT), e a massa média dos frutos. Os efeitos do MBTA variaram de acordo com a concentração aplicada e com a fase fenológica de aplicação. O MBTA, na concentração de $8 \mathrm{mg} \mathrm{L}^{-1}$, aplicado com $25 \%$ das flores abertas, incrementou o teor de sólidos solúveis, a acidez, a quantidade de sólidos solúveis por caixa de $40,8 \mathrm{~kg}$ e a produtividade. Esse mesmo tratamento também reduziu a massa média dos frutos e não alterou o rendimento de suco e o "ratio" do suco dos frutos. O incremento médio foi de 0,49 a $0,65 \%$ na concentração de sólidos solúveis, de 0,11 a $0,13 \mathrm{~kg}$ na quantidade de sólidos solúveis por caixa de 40,8 $\mathrm{kg}$ e de 20,4 kg/planta na produtividade.

Termos para indexação: aminas terciárias; Citrus sp; reguladores vegetais.

\section{EFFECTS OF MBTA [N,N-DIETHYL-2-(4-METYLBENZYLOXY) ETHYLAMINE HYDROCHLORIDE] ON YIELD AND FRUIT QUALITY OF 'PÊRA'SWEET ORANGE (Citrus sinensis L. Osbeck)}

\begin{abstract}
The purpose of this research was to evaluate the effects of MBTA [N,N-diethyl-2-(4-metylbenzyloxy) ethylamine hydrochloride] bioregulator applied on different times and concentrations on the yield and fruit quality of 'Pera' sweet orange. In two consecutive harvest seasons, the MBTA was sprayed in three different concentrations $\left(8 ; 16\right.$ and $\left.32 \mathrm{mg} \mathrm{L}^{-1}\right)$ and in two different phenological phases (25\% and 100\% open flowers) in citrus mature trees, using $7 \mathrm{~L}$ of spray per tree added with Silwett L-77 adjuvant at $0.05 \%$. For each cultivar, twenty fruits per tree were sampled in four different times to determine the soluble solids concentration (SS), titratable acidity (TA), quantity of soluble solids per $40.8 \mathrm{~kg}$ box, juice yield, "ratio" (SS/TA) and average fruit weight. The effects of MBTA varied according to the concentration and phenological phase of application. The MBTA at $8 \mathrm{mg} \mathrm{L}^{-1}$ concentration, sprayed on $25 \%$ of the open flowers, increased the soluble solids concentration, acidity, the quantity of soluble solids per $40.8 \mathrm{~kg}$ box and the yield. The same treatment also decreased the average fruit weight and did not affect the juice yield and the "ratio". The average increase was from 0.49 to $0.65 \%$ on soluble solids concentration, from 0.11 to $0.13 \mathrm{~kg}$ on the quantity of soluble solids per $40.8 \mathrm{~kg}$ box and $20.4 \mathrm{~kg} /$ tree on yield.
\end{abstract}

Index Terms: Tertiary amines; Citrus sp; Plant Growth Regulators; Soluble solids;

\section{INTRODUÇÃO}

O Brasil é o principal produtor mundial de citros, possuindo uma área de produção em torno de 953.000 ha, sendo 836.000 ha ocupados somente por laranjas, onde o Estado de São Paulo é o principal produtor, com $79 \%$ de toda a produção nacional (IBGE, 2004). Desta área de laranjas colhidas, entre 70 a $80 \%$ são destinados à produção de suco de laranja concentrado congelado (SLCC), que gera ao redor de U\$ 1,4 bilhão de receita como resultado de exportação (Visão Agrícola, 2004).

Segundo Wardowski et al. (1995), o teor de sólidos solúveis e a quantidade de suco produzida dos frutos são os fatores utilizados para calcular a quantidade de sólidos solúveis por caixa de laranja produzida. O teor de sólidos solúveis e também de suco do fruto varia de acordo com o estádio de maturação, clima e cultivares, sendo que esses fatores não podem ser controlados pelos produtores. Portanto, técnicas que possam incrementar o teor de sólidos solúveis e a quantidade de suco dos frutos cítricos e que possam ser manipuladas sem a interferência de fatores ambientais, de clima e de solo, são desejáveis para o manejo da cultura dos citros. Dentre essas técnicas, inclui-se a utilização de biorreguladores aplicados

'Trabalho 223-07). Recebido em: 25-09-2007. Aceito para publicação em: 15-05-2008. Parte da tese do primeiro autor, apresentada à ESALQ/USP para obtenção do título de Doutor

${ }^{2}$ Eng. Agr. Dr. E-mail: cmsercil@uol.com.br

${ }^{3}$ Eng. Agr. Dr. Prof. do Depto. de Ciências Biológicas da ESALQ/USP. E-mail: prcastro@esalq.usp.br

${ }^{4}$ Eng. Agr. Dr. Pesquisador Científico da APTA. E-mail: stavares@aptaregional.sp.gov.br

Rev. Bras. Frutic., Jaboticabal - SP, v. 30, n. 3, p.596-603 Setembro 2008 
exogenamente à planta cítrica.

Na citricultura, a utilização de biorreguladores consiste em uma prática cultural importante em diversos países, como Espanha, Estados Unidos e África do Sul. Nesses países, os biorreguladores são utilizados para controlar os processos de florescimento, fixação, crescimento e maturação dos frutos. Alguns dos aspectos relacionados com a maturação dos frutos cítricos são regulados e controlados por hormônios vegetais. Apesar de coincidentes em tempo, a coloração e a maturação interna são processos distintos e regulados, provavelmente, por diferentes substâncias. Sendo assim, atrasar ou adiantar a coloração é um processo relativamente fácil, mas manipular a maturação interna é um processo difícil (Agustí \& Almela, 1991). No entanto, a maioria dos biorreguladores utilizados não altera o conteúdo de suco, o teor de sólidos solúveis ou a acidez dos frutos cítricos (Coggins, 1981; Davies et al., 1997; Serciloto et al., 2003).

O biorregulador MBTA [cloridrato de N,N-dietil-2-(4metilbenziloxi) etilamina], pertencente ao grupo das aminas terciárias, apresenta potencial para utilização comercial em laranjas para processamento. Testes iniciais de campo na Flórida, EUA, realizados com a laranja 'Hamlin', demonstraram aumento significativo no teor de sólidos solúveis na colheita, como resultado de uma única aplicação do produto em pleno florescimento. Ensaios subseqüentes em escala comercial com a laranja 'Hamlin' também foram positivos, assim como para a laranja 'Valência'. No entanto, nesses trabalhos, não foram feitos estudos de outras concentrações e também não foram realizadas análises estatísticas devido à não- aleatorização das parcelas (Campbell et al., 1999).

O conhecimento das técnicas ideais de aplicação dos biorreguladores, bem como dos seus efeitos sobre os parâmetros fisiológicos e de colheita são de fundamental importância para nossas condições edafoclimáticas, estabelecendo, desse modo, as melhores formulações e as cultivares cítricas locais que possam ser submetidas à aplicação dessas substâncias.

O objetivo deste trabalho foi avaliar o efeito de diferentes concentrações e épocas de aplicação do MBTA na qualidade e produtividade da laranja 'Pêra'.

\section{MATERIAL E MÉTODOS}

O experimento foi conduzido em condições de campo, em um pomar comercial, implantado em um Planossolo VermelhoAmarelo, suplementado com irrigação por aspersão em ocasiões de déficit hídrico, localizado na Fazenda Panorama, bairro Mato Seco, no município de Aguaí, Estado de São Paulo, situada a $22,12^{\circ}$ de latitude sul e a $46,98^{\circ}$ de longitude oeste, nas safras de 1999/2000, 2000/2001 e 2001/2002. Avaliou-se o efeito do tratamento da amina terciária MBTA [cloridrato de N,N-dietil-2(4-metilbenziloxi) etilamina] em laranjeira 'Pêra' (Citrus sinensis L. Osbeck). As plantas estavam enxertadas sobre limoeiro 'Cravo' (Citrus limonia L. Osbeck), e o espaçamento entre plantas era de 7,0 x 4,0 metros.

Foram selecionadas árvores homogêneas, com aproximadamente dez anos de idade, quanto ao porte, potencial produtivo, estado fitossanitário e nutricional, sendo que cada árvore constituiu uma parcela. Os tratamentos foram compostos do controle e do MBTA em três diferentes concentrações: $8 \mathrm{mg}$ $\mathrm{L}^{-1} ; 16 \mathrm{mg} \mathrm{L}^{-1}$ e $32 \mathrm{mg} \mathrm{L}^{-1}$, aplicados na fase de $25 \%$ ou na fase de $100 \%$ de flores abertas em cada ano de avaliação.

O biorregulador foi aplicado foliarmente, com o uso de um pulverizador do tipo pistola, sendo gastos aproximadamente 7,0 litros de solução por planta, em toda a extensão da copa. À solução, adicionou-se o adjuvante Silwett L-77 0,05\% com o objetivo de auxiliar a penetração do biorregulador nas folhas e flores das árvores cítricas. As datas de aplicação dos tratamentos foram estabelecidas de acordo com a fenologia das árvores.

Na primeira safra estudada (1999/2000), a aplicação com $25 \%$ de flores abertas foi realizada no dia 12 de agosto de 1999 . A aplicação com $100 \%$ de flores abertas foi realizada no dia 27 de agosto de 1999. Durante a safra de 2000/2001, em função das adversidades climáticas e fitossanitárias, a fixação dos frutos foi afetada, comprometendo a produtividade, preferindo-se não analisar os dados provenientes da mesma, repetindo-se o experimento na safra seguinte (2001/2002). Na segunda safra estudada (2001/2002), as aplicações foram realizadas em 21 de setembro e em 11 de outubro de 2001 para as épocas de $25 \%$ e $100 \%$ de flores abertas, respectivamente.

Em cada safra estudada, foram amostrados 20 frutos no terço médio de cada planta de cada parcela, em quatro diferentes épocas de avaliação. Na safra de 1999/2000, os frutos foram amostrados aos 270; 291; 312 e 333 dias após a antese, sendo a última amostragem realizada no dia 25 de julho de 2000. Na safra de 2001/2002, os frutos foram amostrados aos 229; 250;271 e 292 dias após a antese, sendo a última amostragem realizada no dia 30 de julho de 2002. Em cada época de amostragem, foram avaliados os parâmetros: a) massa dos frutos (g); b) massa do suco $(\mathrm{g})$; c) porcentagem de suco $(\%)$, obtida pela relação massa do suco/massa dos frutos; d) teor de sólidos solúveis (SS) expresso em ${ }^{\circ}$ Brix e obtido através de refratômetro digital (Wardowski et al., 1995; Kimball, 1999); e) acidez titulável (ATT), sendo obtida através de titulação de uma alíquota de $10 \mathrm{~mL}$ de suco com $\mathrm{NaOH} 0,1 \mathrm{~N}$, sendo os resultados expressos em \% de ácido cítrico (Wardowski et al., 1995; Kimball, 1999); f) "ratio" (relação SS/AT), e g) quantidade de sólidos solúveis por caixa de 40,8 kg (kg), determinado segundo Di Giorgi et al. (1990), através da seguinte fórmula: SS ( ${ }^{\circ}$ Brix) x porcentagem de suco (\%) x 40,8 kg (massa da caixa padrão industrial para citros) / 10000. No segundo ano de avaliação, (2002), determinou-se também a produtividade dos frutos produzidos, em kg/planta, na última data de amostragem.

Para os resultados de produtividade, o delineamento experimental utilizado foi o inteiramente casualizado. Para as demais variáveis, o delineamento utilizado foi o inteiramente casualizado, em parcelas subdivididas, em esquema fatorial 7 x 4 , sendo 7 tratamentos e 4 épocas de avaliação, com os tratamentos compondo as parcelas e as épocas de avaliação compondo as subparcelas, onde cada tratamento foi composto de 8 repetições, sendo que cada repetição foi constituída por 20 frutos amostrados em cada planta. Todos os resultados obtidos foram submetidos à análise de variância, sendo que, quando houve significância 
entre os tratamentos, as médias foram comparadas através do teste Duncan, ao nível de 5\% de probabilidade. Os dados de massa média e teor de sólidos solúveis dos frutos foram também submetidos à análise de correlação. Para todas as análises estatísticas, utilizou-se o programa estatístico SAS (SAS Institute, 1989).

\section{RESULTADOS E DISCUSSÃO}

Houve diferenças significativas entre os tratamentos quanto ao teor de sólidos solúveis nos dois anos de avaliação. Em ambos os anos, não houve interação entre os tratamentos e as épocas de avaliação. No ano de 2000, todos os tratamentos foram estatisticamente superiores ao controle, independentemente da época de amostragem dos frutos. Nesse ano, os tratamentos com MBTA nas concentrações de 16 e 32 $\mathrm{mg} \mathrm{L}^{-1}$ aplicados com $100 \%$ de flores abertas apresentaram os maiores teores de sólidos solúveis, sendo também superiores aos tratamentos MBTA 16 e $32 \mathrm{mg} \mathrm{L}^{-1}$ aplicados com $25 \%$ de flores abertas (Tabela 1). Já no ano de 2002, apenas os tratamentos MBTA 8 e $16 \mathrm{mg} \mathrm{L}^{-1}$ aplicados com $25 \%$ de flores abertas superaram estatisticamente o controle, sendo que o maior incremento no teor de sólidos solúveis foi promovido com a concentração de $8 \mathrm{mg} \mathrm{L}^{-1}$ (Tabela 2). Considerando os dois anos avaliados, observa-se uma consistência dos tratamentos MBTA 8 e $16 \mathrm{mg} \mathrm{L}^{-1}$ aplicados com $25 \%$ de flores abertas sobre o teor de sólidos solúveis. Esses dados comprovam o efeito positivo das aminas terciárias no incremento do teor de sólidos solúveis verificados por outros autores (Keithly et al., 1991; Campbell et al., 1999). No entanto, neste trabalho, os resultados positivos sempre foram obtidos com aplicações realizadas com $25 \%$ de flores abertas, o que difere dos dados de Campbell et al. (1999), que obtiveram respostas positivas com $100 \%$ de flores abertas nas cultivares Valência e Hamlin. Em nossas condições e nessa cultivar, também se observou maior resposta ao MBTA quando aplicado na concentração de $8 \mathrm{mg} \mathrm{L}^{-1}$, sendo que, nos Estados Unidos, nas cultivares Valência e Hamlin (Campbell et al., 1999), foram obtidos maiores resultados médios com a concentração de $16 \mathrm{mg} \mathrm{L}^{-1}$. A cultivar Pêra parece ser mais responsiva a substâncias promotoras do desenvolvimento em relação a outras cultivares, já que é uma cultivar que apresenta vários fluxos vegetativos e reprodutivos durante um mesmo ano, sendo que concentrações pequenas podem ser efetivas sobre o metabolismo da planta. Incrementos no teor de sólidos solúveis em tangerinas e laranjas foram obtidos com aplicações do biorregulador etilclozate (Figaron) em experimentos realizados na Espanha (Casas \& Mallent, 1986) e no Japão (Iwahori et al., 1986). O uso do MBTA é mais interessante do ponto de vista produtivo, já que não provoca queda de frutos e folhas, o que pode ocorrer com o uso do Etilclozate em temperaturas elevadas, que também promove redução do vigor das árvores cítricas (Casas \& Lácer, 1989; Hirose et al., 1978).

Houve diferenças significativas entre os tratamentos no rendimento de suco, nos dois anos de avaliação. Em ambos os anos, não houve interação entre os tratamentos e as épocas de avaliação. No ano de 2000 , as plantas-controle $\left(0 \mathrm{mg} \mathrm{L}^{-1}\right)$ tiveram o maior rendimento de suco nos frutos, não diferindo estatisticamente apenas dos tratamentos MBTA $8 \mathrm{mg} \mathrm{L}^{-1}$ aplicado com 25 ou 100\% de flores abertas (Tabela 3). Já, no ano de 2002, apenas os tratamentos MBTA $8 \mathrm{mg} \mathrm{L}^{-1}$ aplicado com $100 \%$ de flores abertas e MBTA $16 \mathrm{mg} \mathrm{L}^{-1}$ aplicado com $25 \%$ de flores abertas foram estatisticamente inferiores ao controle (Tabela 4). Com relação a essa variável, observa-se que somente o tratamento MBTA $8 \mathrm{mg} \mathrm{L}^{-1}$ aplicado com $25 \%$ de flores abertas não diferiu estatisticamente do controle nos dois anos de avaliação.

Houve diferenças significativas entre os tratamentos, na quantidade de sólidos solúveis por caixa de 40,8 kg de frutos, nos dois anos de avaliação. Em ambos os anos avaliados, não houve interação significativa entre os tratamentos e as épocas de avaliação. No ano de 2000, os tratamentos MBTA $8 \mathrm{mg} \mathrm{L}^{-1}$ aplicado com 25 ou $100 \%$ de flores abertas e MBTA $32 \mathrm{mg} \mathrm{L}^{-1}$ aplicado com $100 \%$ de flores abertas superaram, estatisticamente, o controle (Tabela 5). Observa-se que o incremento, na quantidade de sólidos solúveis, produzido por caixa de $40,8 \mathrm{~kg}$, foi somente devido ao incremento no teor de sólidos solúveis (Tabela 1) e não aos incrementos na porcentagem de suco (Tabela 3). Já em 2002, apenas o tratamento MBTA $8 \mathrm{mg} \mathrm{L}^{-1}$ aplicado com $25 \%$ de flores abertas superou, estatisticamente, o controle e os demais tratamentos, produzindo a maior quantidade de sólidos solúveis por caixa de 40,8 $\mathrm{kg}$ (Tabela 6). Esse tratamento também foi o único a superar, estatisticamente, ao controle nos dois anos de avaliação. Embora Campbell et al. (1999) tenham obtido resultados positivos no incremento, no teor de sólidos solúveis em laranjas, em seu trabalho não foi realizado esse tipo de análise, que expressa de melhor forma a produção de sólidos solúveis, o que também poderia contribuir para a efetividade do MBTA no trabalho realizado por Davies et al. (2004), que não obtiveram respostas consistentes com o uso de MBTA em citros, em estudos realizados nos Estados Unidos.

Houve diferenças significativas entre os tratamentos na acidez titulável do suco, nos dois anos de avaliação. Em ambos os anos avaliados, não houve interação entre os tratamentos e as épocas de avaliação. Em 2000, todos os tratamentos incrementaram a acidez do suco, diferindo estatisticamente do controle (Tabela 7). Já no ano de 2002, somente os tratamentos com MBTA $8 \mathrm{mg} \mathrm{L}^{-1}$ aplicado com 25 e $100 \%$ de flores abertas superaram, estatisticamente, o controle, independentemente da época de avaliação (Tabela 8). Observa-se que, tanto em 2000, como em 2002, o incremento no teor de sólidos solúveis promovido pelo MBTAé acompanhado também por um pequeno incremento na acidez, já que os tratamentos que incrementam o teor de sólidos solúveis, também incrementaram os teores de acidez. Parte desse aumento nos teores de sólidos solúveis promovido pelo MBTA deve-se, portanto, a incrementos nos teores de acidez no suco. Apesar de o tratamento MBTA $8 \mathrm{mg} \mathrm{L}^{-1}$ aplicado com $25 \%$ de flores abertas ter incrementado a acidez, o "ratio" do suco não foi alterado por esse tratamento nos dois anos de avaliação (dados não apresentados).

Houve diferenças significativas entre os tratamentos na massa média dos frutos, nos dois anos de avaliação. Em ambos os anos, não houve interação entre os tratamentos e as épocas 
de avaliação. No ano de 2000, todos os tratamentos aplicados com $100 \%$ de flores abertas diminuíram a massa média dos frutos. Os demais tratamentos, aplicados com $25 \%$ de flores abertas, exceto o tratamento MBTA $8 \mathrm{mg} \mathrm{L}^{-1}$, não alteraram a massa média dos frutos em relação ao controle (Tabela 9). Observa-se que os tratamentos que promoveram maiores incrementos nos teores de sólidos solúveis, também diminuíram a massa média dos frutos neste ano de avaliação, o que mostra uma relação inversa entre a massa média dos frutos e teor de sólidos solúveis. Em 2002, não houve interação entre os tratamentos e as épocas de avaliação. Os tratamentos MBTA 8 e $16 \mathrm{mg} \mathrm{L}^{-1}$ aplicados com $25 \%$ de flores abertas e MBTA $8 \mathrm{mg} \mathrm{L}^{-1}$ aplicado com $100 \%$ de flores abertas produziram frutos com massa média inferior aos demais, diferindo estatisticamente do controle (Tabela 10). Os demais tratamentos não alteraram a massa média dos frutos. Observa-se também, neste ano, de acordo com a análise de correlação realizada ( $\mathrm{r}=-$ $\left.0,80^{*}\right)$, que houve relação inversa entre os teores de sólidos solúveis e a massa média dos frutos, já que os tratamentos que incrementaram o teor de sólidos solúveis, produziram frutos com menor massa média. Esse efeito pode estar relacionado a um desvio na partição de assimilados, associado a um incremento na fixação dos frutos promovidos pelo MBTA, já que houve também um incremento na produtividade final das plantas tratadas com o mesmo. A mesma relação inversa entre a massa média do fruto e sua concentração de sólidos solúveis já foi observada por Barry et al. (2004), que notaram que os frutos menores e de menor massa continham maiores teores de sólidos solúveis.
Observa-se pela Figura 1 que os tratamentos MBTA 8 $\mathrm{mg} \mathrm{L}^{-1}$ e $32 \mathrm{mg} \mathrm{L}^{-1}$ aplicados com $25 \%$ de flores abertas apresentaram as maiores produtividades. No entanto, somente o tratamento MBTA $8 \mathrm{mg} \mathrm{L}^{-1}$ aplicado com $25 \%$ de flores abertas superou, estatisticamente, o controle em mais de $20 \mathrm{~kg}$ por planta. Essa maior produtividade está relacionada com um aumento no número de frutos fixados, já que também ocorreu diminuição na massa média dos frutos desse tratamento, nesse mesmo ano (Tabela 10). Alguns biorreguladores, principalmente o ácido giberélico, têm proporcionado incrementos na fixação dos frutos e, conseqüentemente, na produtividade de algumas cultivares cítricas. No entanto, seus efeitos são mais evidentes para tangerinas, principalmente em tangerina 'Clementina' e alguns de seus híbridos (Davies, 1997, apud Sanches, 2000). Com esses resultados obtidos na produtividade e no incremento da quantidade de sólidos solúveis, os efeitos do MBTA devem ser mais bem estudados, já que, em laranjas, as respostas de biorreguladores sobre a produtividade não ocorrem com freqüência, sendo os resultados muito variáveis (Davies, 1997, apud Sanches, 2000; Agustí et al., 1982). Além disso, a maioria dos resultados positivos com o uso de ácido giberélico (Agustí et al., 1982) em mistura ou não com o ácido 2,4diclorofenoxiacético (Koller et al., 1999) e de outros biorreguladores, como a cinetina (Agustí et al., 1990) e o brassinolídeo (Sugyama \& Kuraiashi, 1989), aplicados no início ou em pleno florescimento, na fixação e na produtividade de laranjeiras, são obtidos apenas em cultivares sem sementes.

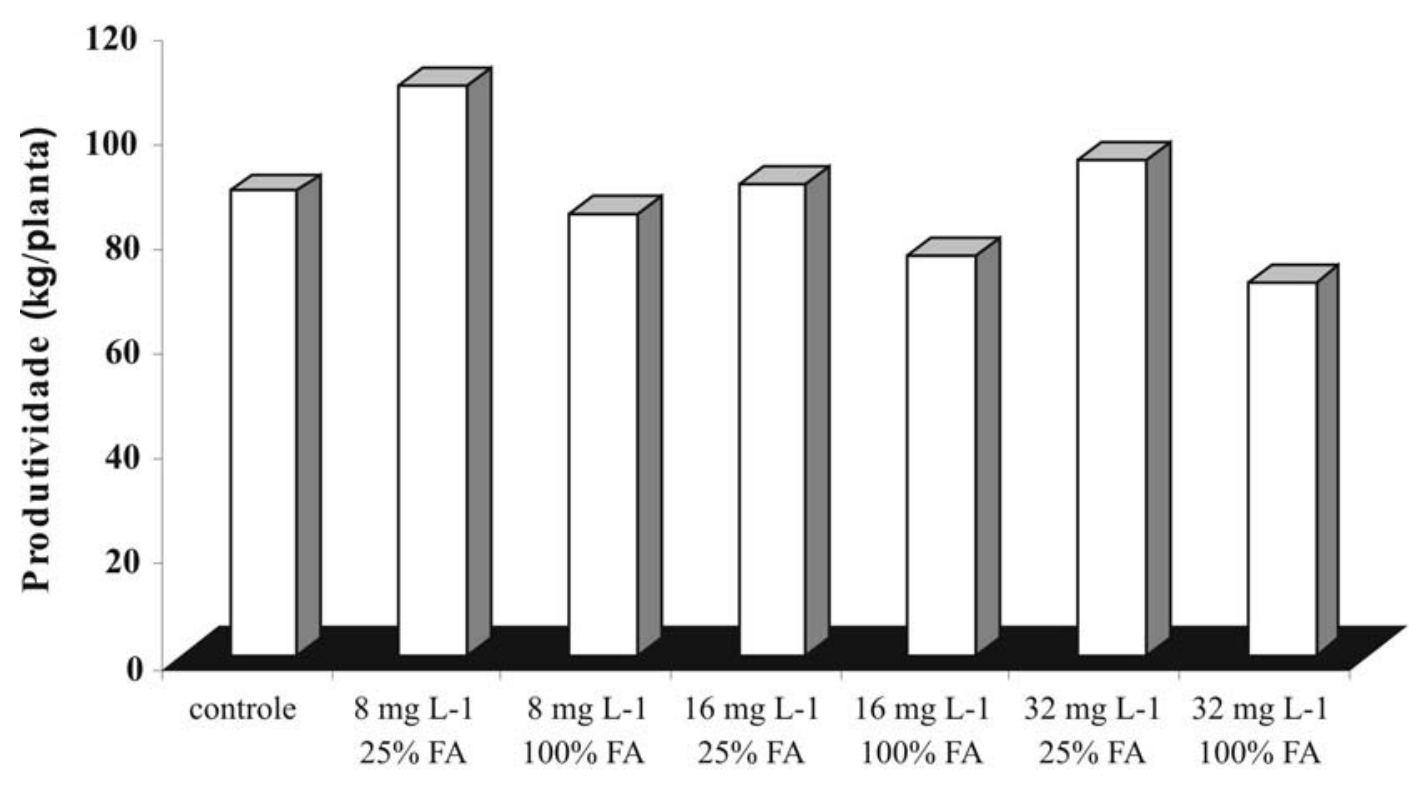

FIGURA 1 - Efeito das diferentes concentrações de MBTA combinadas a diferentes fases de aplicação na produtividade (kg/planta) da laranjeira 'Pêra', no ano de 2002. Obs: letras diferentes indicam diferenças estatísticas, pelo teste Duncan, ao nível de $5 \%$ de probabilidade. FA: flores abertas. 
TABELA 1 - Efeito do MBTA aplicado em diferentes concentrações e fases de aplicação sobre o teor de sólidos solúveis ( ${ }^{\circ}$ Brix) do suco da laranja 'Pêra', no ano de 2000, em quatro diferentes épocas de avaliação.

\begin{tabular}{|c|c|c|c|c|c|c|}
\hline \multirow[b]{2}{*}{$\begin{array}{c}\text { MBTA } \\
\text { (concentrações) }\end{array}$} & \multirow[b]{2}{*}{$\begin{array}{c}\text { Fase de } \\
\text { aplicação }\end{array}$} & \multicolumn{4}{|c|}{ Dias após a antese } & \multirow[b]{2}{*}{$\begin{array}{l}\text { Médias dos } \\
\text { tratamentos }\end{array}$} \\
\hline & & 270 & 291 & 312 & 333 & \\
\hline $0 \mathrm{mg} \mathrm{L}^{-1}$ & & 9,67 & 9,91 & 10,45 & 10,22 & $10,07 \mathrm{~d}$ \\
\hline $8 \mathrm{mg} \mathrm{L}^{-1}$ & $25 \%$ F.A. $^{1}$ & 10,35 & 10,42 & 11,04 & 11,11 & $10,72 \mathrm{abc}$ \\
\hline $8 \mathrm{mg} \mathrm{L}^{-1}$ & $100 \%$ F.A. & 10,31 & 10,66 & 10,93 & 11,02 & $10,74 \mathrm{abc}$ \\
\hline $16 \mathrm{mg} \mathrm{L}^{-1}$ & $25 \%$ F.A. & 10,14 & 10,53 & 11,04 & 10,70 & $10,60 \mathrm{c}$ \\
\hline $16 \mathrm{mg} \mathrm{L}^{-1}$ & $100 \%$ F.A. & 10,41 & 10,90 & 11,23 & 11,26 & $10,94 \mathrm{a}$ \\
\hline $32 \mathrm{mg} \mathrm{L}^{-1}$ & $25 \%$ F.A. & 10,37 & 10,45 & 10,85 & 10,81 & $10,63 \mathrm{bc}$ \\
\hline $32 \mathrm{mg} \mathrm{L}^{-1}$ & $100 \%$ F.A. & 10,46 & 10,71 & 11,22 & 11,24 & $10,91 \mathrm{a}$ \\
\hline Médias das época & & $10,25 \mathrm{C}$ & $10,51 \mathrm{~B}$ & $10,96 \mathrm{~A}$ & $10,91 \mathrm{~A}$ & \\
\hline
\end{tabular}

Médias seguidas de mesma letra minúscula na coluna e maiúscula na linha não diferem significativamente, pelo teste Duncan, ao nível de 5\% de probabilidade. ${ }^{1}$ F.A.: Flores abertas.

TABELA 2 - Efeito do MBTA aplicado em diferentes concentrações e fases de aplicação sobre o teor de sólidos solúveis ( ${ }^{\circ}$ Brix) do suco da laranja 'Pêra', no ano de 2002, em quatro diferentes épocas de avaliação.

\begin{tabular}{|c|c|c|c|c|c|c|}
\hline \multirow[b]{2}{*}{$\begin{array}{c}\text { MBTA } \\
\text { (concentrações) }\end{array}$} & \multirow[b]{2}{*}{$\begin{array}{c}\text { Fase de } \\
\text { aplicação }\end{array}$} & \multicolumn{4}{|c|}{ Dias após a antese } & \multirow[b]{2}{*}{$\begin{array}{l}\text { Médias dos } \\
\text { tratamentos }\end{array}$} \\
\hline & & 229 & 250 & 271 & 292 & \\
\hline $0 \mathrm{mg} \mathrm{L}^{-1}$ & & 9,44 & 10,32 & 10,85 & 10,11 & $10,14 \mathrm{~cd}$ \\
\hline $8 \mathrm{mg} \mathrm{L}^{-1}$ & $25 \%$ F.A. $^{1}$ & 9,69 & 10,80 & 11,27 & 10,84 & $10,63 \mathrm{a}$ \\
\hline $8 \mathrm{mg} \mathrm{L}^{-1}$ & $100 \%$ F.A. & 9,25 & 10,20 & 10,76 & 10,71 & $10,22 \mathrm{bc}$ \\
\hline $16 \mathrm{mg} \mathrm{L}^{-1}$ & $25 \%$ F.A. & 9,54 & 10,49 & 11,15 & 10,61 & $10,44 \mathrm{ab}$ \\
\hline $16 \mathrm{mg} \mathrm{L}^{-1}$ & $100 \%$ F.A. & 9,27 & 9,94 & 10,39 & 10,27 & 9,96 d \\
\hline $32 \mathrm{mg} \mathrm{L}^{-1}$ & $25 \%$ F.A. & 9,29 & 9,91 & 10,30 & 10,17 & $9,91 \mathrm{~d}$ \\
\hline $32 \mathrm{mg} \mathrm{L}^{-1}$ & $100 \%$ F.A. & 9,14 & 9,96 & 10,41 & 10,13 & $9,91 \mathrm{~d}$ \\
\hline Médias das época & & $9,38 \mathrm{C}$ & $10,25 \mathrm{~B}$ & $10,72 \mathrm{~A}$ & $10,41 \mathrm{~B}$ & \\
\hline
\end{tabular}

Médias seguidas de mesma letra minúscula na coluna e maiúscula na linha não diferem significativamente, pelo teste Duncan, ao nível de 5\% de probabilidade. ${ }^{1}$ F.A.: Flores abertas.

TABELA 3 - Efeito do MBTA aplicado em diferentes concentrações e fases de aplicação sobre o rendimento de suco (\%) da laranja 'Pêra' no ano de 2000 em quatro diferentes épocas de avaliação.

\begin{tabular}{|c|c|c|c|c|c|c|}
\hline \multirow[b]{2}{*}{$\begin{array}{c}\text { MBTA } \\
\text { (concentrações) }\end{array}$} & \multirow[b]{2}{*}{$\begin{array}{c}\text { Fase de } \\
\text { aplicação }\end{array}$} & \multicolumn{4}{|c|}{ Dias após a antese } & \multirow[b]{2}{*}{$\begin{array}{l}\text { Médias dos } \\
\text { tratamentos }\end{array}$} \\
\hline & & 270 & 291 & 312 & 333 & \\
\hline $0 \mathrm{mg} \mathrm{L}^{-1}$ & & 56,46 & 55,23 & 55,52 & 57,47 & $56,20 \mathrm{a}$ \\
\hline $8 \mathrm{mg} \mathrm{L}^{-1}$ & $25 \%$ F.A. $^{1}$ & 54,51 & 55,39 & 53,49 & 57,19 & $55,22 \mathrm{ab}$ \\
\hline $8 \mathrm{mg} \mathrm{L}^{-1}$ & $100 \%$ F.A. & 53,13 & 55,78 & 54,31 & 57,20 & $55,11 \mathrm{ab}$ \\
\hline $16 \mathrm{mg} \mathrm{L}^{-1}$ & $25 \%$ F.A. & 50,58 & 52,56 & 52,98 & 55,14 & $52,74 \mathrm{~d}$ \\
\hline $16 \mathrm{mg} \mathrm{L}^{-1}$ & $100 \%$ F.A. & 51,84 & 52,74 & 53,61 & 55,87 & $53,44 \mathrm{~cd}$ \\
\hline $32 \mathrm{mg} \mathrm{L}^{-1}$ & $25 \%$ F.A. & 52,70 & 53,04 & 51,67 & 54,58 & $52,95 \mathrm{~cd}$ \\
\hline $32 \mathrm{mg} \mathrm{L}^{-1}$ & $100 \%$ F.A. & 52,83 & 52,65 & 54,49 & 57,07 & $54,25 \mathrm{bc}$ \\
\hline Médias das épocas & & $53,13 \mathrm{~B}$ & $53,83 \mathrm{~B}$ & $53,71 \mathrm{~B}$ & $56,41 \mathrm{~A}$ & \\
\hline
\end{tabular}

Médias seguidas de mesma letra minúscula na coluna e maiúscula na linha não diferem significativamente, pelo teste Duncan, ao nível de 5\% de probabilidade. ${ }^{1}$ F.A.: Flores abertas.

TABELA 4 - Efeito do MBTA aplicado em diferentes concentrações e fases de aplicação sobre o rendimento de suco (\%) da laranja 'Pêra', no ano de 2002, em quatro diferentes épocas de avaliação.

\begin{tabular}{lcccccc}
\hline & & \multicolumn{5}{c}{ Dias após a antese } \\
\cline { 3 - 6 } $\begin{array}{c}\text { MBTA } \\
\text { (concentrações) }\end{array}$ & $\begin{array}{c}\text { Fase de } \\
\text { aplicação }\end{array}$ & $\mathbf{2 2 9}$ & $\mathbf{2 5 0}$ & $\mathbf{2 7 1}$ & $\mathbf{2 9 2}$ & $\begin{array}{c}\text { Médias dos } \\
\text { tratamentos }\end{array}$ \\
\hline $0 \mathrm{mg} \mathrm{L}^{-1}$ & & 42,88 & 55,39 & 54,57 & 56,15 & $52,36 \mathrm{a}$ \\
$8 \mathrm{mg} \mathrm{L}^{-1}$ & $25 \%$ F.A. & 42,73 & 55,47 & 52,92 & 57,22 & $52,39 \mathrm{a}$ \\
$8 \mathrm{mg} \mathrm{L}^{-1}$ & $100 \%$ F.A. & 38,16 & 53,04 & 49,45 & 54,11 & $48,90 \mathrm{c}$ \\
$16 \mathrm{mg} \mathrm{L}^{-1}$ & $25 \%$ F.A. & 38,90 & 53,97 & 51,76 & 52,79 & $49,59 \mathrm{bc}$ \\
$16 \mathrm{mg} \mathrm{L}^{-1}$ & $100 \%$ F.A. & 45,21 & 55,65 & 52,73 & 58,52 & $53,11 \mathrm{a}$ \\
$32 \mathrm{mg} \mathrm{L}^{-1}$ & $25 \%$ F.A. & 44,54 & 55,27 & 52,89 & 53,54 & $51,45 \mathrm{ab}$ \\
$32 \mathrm{mg} \mathrm{L}^{-1}$ & $100 \%$ F.A. & 45,35 & 54,11 & 52,04 & 54,61 & $51,51 \mathrm{ab}$ \\
\hline Médias das épocas & & $42,63 \mathrm{C}$ & $54,73 \mathrm{~A}$ & $52,29 \mathrm{~B}$ & $55,28 \mathrm{~A}$ & \\
\hline
\end{tabular}

Médias seguidas de mesma letra minúscula na coluna e maiúscula na linha não diferem significativamente, pelo teste Duncan, ao nível de 5\% de probabilidade. ${ }^{1}$ F.A.: Flores abertas. 
TABELA 5 - Efeito do MBTA aplicado em diferentes concentrações e fases de aplicação sobre a quantidade de sólidos solúveis (kg) por caixa de 40,8 kg de frutos, no ano de 2000, em quatro diferentes épocas de avaliação.

\begin{tabular}{|c|c|c|c|c|c|c|}
\hline \multirow[b]{2}{*}{$\begin{array}{c}\text { MBTA } \\
\text { (concentrações) }\end{array}$} & \multirow[b]{2}{*}{$\begin{array}{c}\text { Fase de } \\
\text { aplicação }\end{array}$} & \multicolumn{4}{|c|}{ Dias após a antese } & \multirow[b]{2}{*}{$\begin{array}{l}\text { Médias dos } \\
\text { tratamentos }\end{array}$} \\
\hline & & 270 & 291 & 312 & 333 & \\
\hline $0 \mathrm{mg} \mathrm{L}^{-1}$ & & 2,23 & 2,23 & 2,37 & 2,40 & $2,31 \mathrm{~b}$ \\
\hline $8 \mathrm{mg} \mathrm{L}^{-1}$ & $25 \%$ F.A. $^{1}$ & 2,35 & 2,37 & 2,41 & 2,60 & $2,44 \mathrm{a}$ \\
\hline $8 \mathrm{mg} \mathrm{L}^{-1}$ & $100 \%$ F.A. & 2,21 & 2,43 & 2,42 & 2,54 & $2,39 \mathrm{a}$ \\
\hline $16 \mathrm{mg} \mathrm{L}^{-1}$ & $25 \%$ F.A. & 2,10 & 2,25 & 2,40 & 2,40 & $2,28 \mathrm{~b}$ \\
\hline $16 \mathrm{mg} \mathrm{L}^{-1}$ & $100 \%$ F.A. & 2,20 & 2,31 & 2,45 & 2,57 & $2,37 \mathrm{ab}$ \\
\hline $32 \mathrm{mg} \mathrm{L}^{-1}$ & $25 \%$ F.A. & 2,24 & 2,26 & 2,27 & 2,42 & $2,30 \mathrm{~b}$ \\
\hline $32 \mathrm{mg} \mathrm{L}^{-1}$ & $100 \%$ F.A. & 2,25 & 2,30 & 2,47 & 2,62 & $2,41 \mathrm{a}$ \\
\hline Médias das épocas & & $2,22 \mathrm{D}$ & $2,31 \mathrm{C}$ & $2,40 \mathrm{~B}$ & $2,51 \mathrm{~A}$ & \\
\hline
\end{tabular}

Médias seguidas de mesma letra minúscula na coluna e maiúscula na linha não diferem significativamente, pelo teste Duncan, ao nível de 5\% de probabilidade. ${ }^{1}$ F.A.: Flores abertas.

TABELA 6 - Efeito do MBTA aplicado em diferentes concentrações e fases de aplicação sobre a quantidade de sólidos solúveis (kg) por caixa de 40,8 kg de frutos, no ano de 2002, em quatro diferentes épocas de avaliação.

\begin{tabular}{lcccccc}
\hline & & \multicolumn{4}{c}{ Dias após a antese } & Médias dos \\
\cline { 3 - 5 } $\begin{array}{c}\text { MBTA } \\
\text { (concentrações) }\end{array}$ & $\begin{array}{c}\text { Fase de } \\
\text { aplicação }\end{array}$ & $\mathbf{2 2 9}$ & $\mathbf{2 5 0}$ & $\mathbf{2 7 1}$ & $\mathbf{2 9 2}$ & $\begin{array}{c}\text { Ménentos } \\
\text { tratamentos }\end{array}$ \\
\hline $0 \mathrm{mg} \mathrm{L}^{-1}$ & & 1,65 & 2,34 & 2,40 & 2,33 & $2,18 \mathrm{~b}$ \\
$8 \mathrm{mg} \mathrm{L}^{-1}$ & $25 \%$ F.A. & 1,69 & 2,45 & 2,41 & 2,53 & $2,29 \mathrm{a}$ \\
$8 \mathrm{mg} \mathrm{L}^{-1}$ & $100 \%$ F.A. & 1,39 & 2,20 & 2,17 & 2,39 & $2,04 \mathrm{~d}$ \\
$16 \mathrm{mg} \mathrm{L}^{-1}$ & $25 \%$ F.A. & 1,51 & 2,31 & 2,35 & 2,28 & $2,12 \mathrm{bcd}$ \\
$16 \mathrm{mg} \mathrm{L}^{-1}$ & $100 \%$ F.A. & 1,72 & 2,26 & 2,24 & 2,42 & $2,15 \mathrm{bc}$ \\
$32 \mathrm{mg} \mathrm{L}^{-1}$ & $25 \%$ F.A. & 1,69 & 2,23 & 2,23 & 2,22 & $2,07 \mathrm{~cd}$ \\
$32 \mathrm{mg} \mathrm{L}^{-1}$ & $100 \%$ F.A. & 1,65 & 2,20 & 2,21 & 2,27 & $2,08 \mathrm{bcd}$ \\
\hline Médias das épocas & & $1,61 \mathrm{~B}$ & $2,29 \mathrm{~A}$ & $2,28 \mathrm{~A}$ & $2,36 \mathrm{~A}$ & \\
\hline
\end{tabular}

Médias seguidas de mesma letra minúscula na coluna e maiúscula na linha não diferem significativamente, pelo teste Duncan, ao nível de 5\% de probabilidade. ${ }^{1}$ F.A.: Flores abertas.

TABELA 7 - Efeito do MBTA aplicado em diferentes concentrações e fases de aplicação sobre a porcentagem de acidez titulável (\%) do suco da laranja 'Pêra', no ano de 2000, em quatro diferentes épocas de avaliação

\begin{tabular}{|c|c|c|c|c|c|c|}
\hline \multirow[b]{2}{*}{$\begin{array}{c}\text { MBTA } \\
\text { (concentrações) }\end{array}$} & \multirow[b]{2}{*}{$\begin{array}{c}\text { Fase de } \\
\text { aplicação }\end{array}$} & \multicolumn{4}{|c|}{ Dias após a antese } & \multirow[b]{2}{*}{$\begin{array}{l}\text { Médias dos } \\
\text { tratamentos }\end{array}$} \\
\hline & & 270 & 291 & 312 & 333 & \\
\hline $0 \mathrm{mg} \mathrm{L}^{-1}$ & & 1,25 & 1,09 & 1,08 & 1,07 & $1,12 \mathrm{~d}$ \\
\hline $8 \mathrm{mg} \mathrm{L}^{-1}$ & $25 \%$ F.A. $^{1}$ & 1,43 & 1,17 & 1,16 & 1,05 & $1,20 \mathrm{c}$ \\
\hline $8 \mathrm{mg} \mathrm{L}^{-1}$ & $100 \%$ F.A. & 1,34 & 1,30 & 1,24 & 1,20 & $1,27 \mathrm{bc}$ \\
\hline $16 \mathrm{mg} \mathrm{L}^{-1}$ & $25 \%$ F.A. & 1,61 & 1,47 & 1,38 & 1,28 & $1,43 \mathrm{a}$ \\
\hline $16 \mathrm{mg} \mathrm{L}^{-1}$ & $100 \%$ F.A. & 1,52 & 1,34 & 1,24 & 1,18 & $1,32 \mathrm{~b}$ \\
\hline $32 \mathrm{mg} \mathrm{L}^{-1}$ & $25 \%$ F.A. & 1,47 & 1,30 & 1,27 & 1,15 & $1,30 \mathrm{~b}$ \\
\hline $32 \mathrm{mg} \mathrm{L}^{-1}$ & $100 \%$ F.A. & 1,40 & 1,28 & 1,24 & 1,11 & $1,26 \mathrm{bc}$ \\
\hline
\end{tabular}

Médias seguidas de mesma letra minúscula na coluna e maiúscula na linha não diferem significativamente, pelo teste Duncan, ao nível de 5\% de probabilidade. ${ }^{1}$ F.A.: Flores abertas.

TABELA 8 - Efeito do MBTA aplicado em diferentes concentrações e fases de aplicação sobre a porcentagem de acidez titulável (\%) do suco da laranja 'Pêra', no ano de 2002, em quatro diferentes épocas de avaliação.

\begin{tabular}{|c|c|c|c|c|c|c|}
\hline \multirow[b]{2}{*}{$\begin{array}{c}\text { MBTA } \\
\text { (concentrações) }\end{array}$} & \multirow[b]{2}{*}{$\begin{array}{c}\text { Fase de } \\
\text { aplicação }\end{array}$} & \multicolumn{4}{|c|}{ Dias após a antese } & \multirow[b]{2}{*}{$\begin{array}{l}\text { Médias dos } \\
\text { tratamentos }\end{array}$} \\
\hline & & 229 & 250 & 271 & 292 & \\
\hline $0 \mathrm{mg} \mathrm{L}^{-1}$ & & 1,43 & 1,21 & 1,15 & 0,93 & $1,18 \mathrm{~cd}$ \\
\hline $8 \mathrm{mg} \mathrm{L}^{-1}$ & $25 \%$ F.A. ${ }^{1}$ & 1,55 & 1,28 & 1,25 & 1,06 & $1,28 \mathrm{~b}$ \\
\hline $8 \mathrm{mg} \mathrm{L}^{-1}$ & $100 \%$ F.A. & 1,60 & 1,35 & 1,38 & 1,12 & $1,36 \mathrm{a}$ \\
\hline $16 \mathrm{mg} \mathrm{L}^{-1}$ & $25 \%$ F.A. & 1,48 & 1,24 & 1,24 & 1,02 & $1,24 \mathrm{bc}$ \\
\hline $16 \mathrm{mg} \mathrm{L}^{-1}$ & $100 \%$ F.A. & 1,45 & 1,17 & 1,11 & 0,97 & $1,18 \mathrm{~d}$ \\
\hline $32 \mathrm{mg} \mathrm{L}^{-1}$ & $25 \%$ F.A. & 1,46 & 1,16 & 1,13 & 0,97 & $1,18 \mathrm{~cd}$ \\
\hline $32 \mathrm{mg} \mathrm{L}^{-1}$ & $100 \%$ F.A. & 1,43 & 1,16 & 1,15 & 1,00 & $1,19 \mathrm{~cd}$ \\
\hline Médias das época & & $1,49 \mathrm{~A}$ & $1,22 \mathrm{~B}$ & $1,20 \mathrm{~B}$ & $1,01 \mathrm{C}$ & \\
\hline
\end{tabular}

Médias seguidas de mesma letra minúscula na coluna e maiúscula na linha não diferem significativamente, pelo teste Duncan, ao nível de 5\% de probabilidade. ${ }^{1}$ F.A.: Flores abertas. 
TABELA 9 - Efeito do MBTA aplicado em diferentes concentrações e fases de aplicação sobre a massa média (g) dos frutos, no ano de 2000 , em quatro diferentes épocas de avaliação.

\begin{tabular}{|c|c|c|c|c|c|c|}
\hline \multirow[b]{2}{*}{$\begin{array}{c}\text { MBTA } \\
\text { (concentrações) }\end{array}$} & \multirow[b]{2}{*}{$\begin{array}{c}\text { Fase de } \\
\text { aplicação }\end{array}$} & \multicolumn{4}{|c|}{ Dias após a antese } & \multirow[b]{2}{*}{$\begin{array}{l}\text { Médias dos } \\
\text { tratamentos }\end{array}$} \\
\hline & & 270 & 291 & 312 & 333 & \\
\hline $0 \mathrm{mg} \mathrm{L}^{-1}$ & & 148,1 & 151,0 & 148,1 & 149,8 & $149,2 \mathrm{a}$ \\
\hline $8 \mathrm{mg} \mathrm{L}^{-1}$ & $25 \%$ F.A. $^{1}$ & 134,9 & 145,5 & 147,5 & 148,3 & $144,0 \mathrm{~b}$ \\
\hline $8 \mathrm{mg} \mathrm{L}^{-1}$ & $100 \%$ F.A. & 135,4 & 141,8 & 147,6 & 148,1 & $143,2 \mathrm{~b}$ \\
\hline $16 \mathrm{mg} \mathrm{L}^{-1}$ & $25 \%$ F.A. & 140,6 & 149,6 & 155,4 & 164,0 & $152,4 \mathrm{a}$ \\
\hline $16 \mathrm{mg} \mathrm{L}^{-1}$ & $100 \%$ F.A. & 131,5 & 139,2 & 147,5 & 149,4 & $141,9 \mathrm{~b}$ \\
\hline $32 \mathrm{mg} \mathrm{L}^{-1}$ & $25 \%$ F.A. & 137,9 & 159,5 & 156,8 & 163,3 & $154,2 \mathrm{a}$ \\
\hline $32 \mathrm{mg} \mathrm{L}^{-1}$ & $100 \%$ F.A. & 133,2 & 141,2 & 140,7 & 143,0 & $139,7 \mathrm{~b}$ \\
\hline Médias das época & & $137,5 \mathrm{C}$ & $146,7 \mathrm{~B}$ & $149,8 \mathrm{~B}$ & $154,3 \mathrm{~A}$ & \\
\hline
\end{tabular}

Médias seguidas de mesma letra minúscula na coluna e maiúscula na linha não diferem significativamente, pelo teste Duncan, ao nível de 5\% de probabilidade. ${ }^{1}$ F.A.: Flores abertas.

TABELA 10 - Efeito do MBTA aplicado em diferentes concentrações e fases de aplicação sobre a massa média (g) dos frutos, no ano de 2002, em quatro diferentes épocas de avaliação.

\begin{tabular}{|c|c|c|c|c|c|c|}
\hline \multirow[b]{2}{*}{$\begin{array}{c}\text { MBTA } \\
\text { (concentrações) }\end{array}$} & \multirow[b]{2}{*}{$\begin{array}{c}\text { Fase de } \\
\text { aplicação }\end{array}$} & \multicolumn{4}{|c|}{ Dias após a antese } & \multirow[b]{2}{*}{$\begin{array}{l}\text { Médias dos } \\
\text { tratamentos }\end{array}$} \\
\hline & & 229 & 250 & 271 & 292 & \\
\hline $0 \mathrm{mg} \mathrm{L}^{-1}$ & & 140,9 & 147,6 & 142,6 & 165,3 & $149,6 \mathrm{ab}$ \\
\hline $8 \mathrm{mg} \mathrm{L}^{-1}$ & $25 \%$ F.A. $^{1}$ & 136,5 & 134,8 & 135,0 & 152,2 & $139,6 \mathrm{~d}$ \\
\hline $8 \mathrm{mg} \mathrm{L}^{-1}$ & $100 \%$ F.A. & 137,0 & 142,2 & 137,7 & 151,9 & $142,2 \mathrm{~cd}$ \\
\hline $16 \mathrm{mg} \mathrm{L}^{-1}$ & $25 \%$ F.A. & 138,1 & 140,8 & 137,5 & 152,9 & $142,5 \mathrm{~cd}$ \\
\hline $16 \mathrm{mg} \mathrm{L}^{-1}$ & $100 \%$ F.A. & 144,3 & 153,6 & 149,4 & 158,9 & $151,5 \mathrm{a}$ \\
\hline $32 \mathrm{mg} \mathrm{L}^{-1}$ & $25 \%$ F.A. & 140,9 & 151,2 & 153,0 & 160,2 & $151,3 \mathrm{a}$ \\
\hline $32 \mathrm{mg} \mathrm{L}^{-1}$ & $100 \%$ F.A. & 137,7 & 142,3 & 142,6 & 157,0 & $145,0 \mathrm{bc}$ \\
\hline Médias das épocas & & $139,3 \mathrm{C}$ & $144,5 \mathrm{~B}$ & $142,5 \mathrm{BC}$ & $156,9 \mathrm{~A}$ & \\
\hline
\end{tabular}

Médias seguidas de mesma letra minúscula na coluna e maiúscula na linha não diferem significativamente, pelo teste Duncan, ao nível de 5\% de probabilidade. ${ }^{1}$ F.A.: Flores abertas.

\section{CONCLUSÕES}

1- A aplicação do MBTA incrementou a produtividade e a qualidade da laranja 'Pêra' nas condições brasileiras, sendo esse efeito dependente da época e da concentração aplicada.

2- O MBTA na concentração de $8 \mathrm{mg} \mathrm{L}^{-1}$ aplicado com $25 \%$ de flores abertas incrementa o teor de sólidos solúveis, a acidez titulável e a quantidade de sólidos solúveis por caixa de $40,8 \mathrm{~kg}$ de frutos. Esse mesmo tratamento reduz a massa média dos frutos e não promove alterações no "ratio" e no rendimento de suco dos frutos da laranjeira 'Pêra'.

3- A produtividade é incrementada com a aplicação de MBTA na concentração de $8 \mathrm{mg} \mathrm{L}^{-1}$ quando as plantas apresentam $25 \%$ de flores abertas.

\section{REFERÊNCIAS}

AGUSTÍ, M.; GARCÍA-MARÍ, F.; GUARDIOLA, J.L. Gibberellic acid and fruit-set in sweet orange. Scientia Horticulturae, Amsterdam, v.17, p.257-264, 1982.

AGUSTÍ, M.; ALMELA,V.; MINGO-CASTEL, A.M. Efecto de la quinetina y el rayado sobre el cuajado de la variedad de naranjo 'Navelate' [Citrus sinensis (L.) Osbeck]. Investigación Agrária, Madrid, v.5, n.1, 1990.
AGUSTÍ, M.; ALMELA, V. Aplicación de fitorreguladores en citricultura. Barcelona: Aedos, 1991. 261p.

BARRY, G.H.; CASTLE, W.S; DAVIES, F.S. Soluble solids accumulation in 'Valencia' sweet orange as related to rootstock selection and fruit size. Journal of American Society for Horticultural Science, Geneva, v.129, n.4, p.594-598, 2004.

CAMPBELL, C.A.; TAGGART, T.; KEITHLY, J. A novel plant growth regulator, MBTA, increases soluble solids (brix) of 'Valencia' orange. Proceedings of the Florida State Horticultural Science, Tallahasse, v. 112, p.25-28, 1999.

CASAS, A.; LLÁCER, J. El color de los frutos cítricos. Modificación del color mediante tratamientos pre-recolección. Revista Agroquimica y Tecnologia de Alimentos, Valencia, v. 29, n.2, p. 173-190, 1989

CASAS, A.; MALLENT, D. Efectos del figaron sobre la calidad de las naranjas Navellina y de las mandarinas Satsuma. Revista Agroquimica y Tecnologia de Alimentos, Valencia, v.26, n.2, p. 239-248, 1986.

COGGINS Jr., C.W.; The influence of exogenous growth regulators on rind quality and internal quality of citrus fruits. Proceedings of the International Society of Citriculture, Tokyo, v.1, p.214216, 1981. 
DAVIES, F.S.; CAMPBELL, C.A.; ZALMAN, G.R. Gibberelic acid sprays for improving peel quality and increasing juice yield of processing oranges. Proceedings of Florida State Horticultural Society, Tallahasse, v.110, p.16-21, 1997.

DAVIES, F.S.; STOVER, E.;ZALMAN, G.R.; CILIENTO, S. Ecolyst application timing and juice corrected soluble solids (brix) of processing oranges and grapefruit in Florida. Horttechnology, Alexandria, v.14, n.2, p.207-212, 2004

DI GIORGI, F; IDE, B.Y.; DIB, K. et al. Contribuição ao estudo do comportamento de algumas variedades de citros e suas implicações agroindustriais. Laranja, Cordeirópolis, v.11, n.2, p.567-612, 1990.

HIROSE, K.; IWAHORI, I.; SUZUKI, K. IZZA (5-chloroindazol-8acetic acid ethyl ester) as a new thinning agent Satsuma mandarin (Citrus unshiu Marc.). Proceedings of International Society of Citriculture, Sydney, v.1, p. 270-273, 1978.

IBGE - Instituto Brasileiro de Geografia e Estatística. Produção Agrícola Municipal (PAM) - estatísticas de 2003. Disponível em: <http://www.sidra.ibge.gov.br.>

IWAHORI, S.; TOMINAGA, S.; OOHATA, J.T. Ethylclozate accelerates colouration and enhances fruit quality of Ponkan, Citrus reticulata Blanco. Scientia Horticulturae, Amsterdam, v.28, p.243-250, 1986.

KEITHLY, J.H.; YOKOYAMA, H.; GAUSMAN, H.W. Regulation of crop growth and yield by tertiary amine bioregulators. In: GAUSMAN, H.W. (Ed.). Plant biochemical regulators. New York: Marcel Dekker, 1991. p.233-246.
KIMBALL, D.A. Citrus processing: a complete guide. $2^{\text {nd }}$ ed. Gaithersburg: Aspen Publications, 1999.

KOLLER, O.C.; SCHAFFER, G.; SARTORI, I.A.; LIMA, J.G.Efeito da anelagem , fitorreguladores e fungicidas sobre a fixação de frutos na laranjeira 'Monte Parnaso'. Revista Brasileira de Fruticultura, Jaboticabal, v.21, n.1, p.70-73, 1999.

SANCHES, F.R. Aplicação de biorreguladores vegetais: aspectos fisiológicos e aplicações práticas na citricultura mundial. Jaboticabal: FUNEP, 2000. 160p.

SAS INSTITUTE. Propierty software: release 6.08. Cary: Statistical Analysis System Institute, 1989.

SERCILOTO, C.M.; CASTRO, P.R.C.; TAVARES, S.; MEDINA, C.L. Desbaste e desenvolvimento do tangor 'Murcott' com o uso de biorreguladores. Laranja, Cordeirópolis, v.24, n.1, p.95-111, 2003.

VISÃO AGRÍCOLA. Citrus. Piracicaba: ESALQ, n.1, dez. 1994, $135 \mathrm{p}$.

WARDOWSKI, W.; WHIGHAM, J.; GRIERSON, W.; SOULE, J. Quality tests for Florida citrus. Gainesville: University of Florida, 1995. 17 p. (Cooperative Extension Bulletin, SP99.) 